BNL-NT-04/14

RBRC-414

hep-ph/0404240

November 5, 2018

\title{
Perturbative generation of a strange-quark asymmetry in the nucleon
}

\author{
Stefano Catani ${ }^{(a) *}$, Daniel de Florian ${ }^{(b) \dagger}$, Germán Rodrigo ${ }^{(c) \ddagger}$, \\ and Werner Vogelsang ${ }^{(d) \S}$ \\ ${ }^{(a)}$ INFN, Sezione di Firenze and Dipartimento di Fisica, Università di Firenze, \\ I-50019 Sesto Fiorentino, Florence, Italy \\ ${ }^{(b)}$ Departamento de Física, FCEYN, Universidad de Buenos Aires, \\ (1428) Pabellón 1 Ciudad Universitaria, Capital Federal, Argentina \\ ${ }^{(c)}$ Instituto de Física Corpuscular, Apartado de Correos 22085, E-46071 Valencia, Spain \\ ${ }^{(d)}$ Physics Department and RIKEN-BNL Research Center, \\ Brookhaven National Laboratory, Upton, NY 11973, U.S.A.
}

\begin{abstract}
We point out that perturbative evolution in QCD at three loops generates a strangeantistrange asymmetry $s(x)-\bar{s}(x)$ in the nucleon's sea just from the fact that the nucleon has non-vanishing up and down quark valence densities. The recently computed three-loop splitting functions allow for an estimate of this effect. We find that a fairly sizable asymmetry may be generated. Results for analogous asymmetries in the heavy-quark sector are also presented.
\end{abstract}

\footnotetext{
*E-mail: stefano.catani@fi.infn.it

†E-mail: deflo@df.uba.ar

†E-mail: german.rodrigo@cern.ch

§E-mail: vogelsan@quark.phy.bnl.gov
} 


\section{Introduction}

Strange quarks and antiquarks play a fundamental role in the structure of the nucleon [1]. Among the various strangeness-related properties of the nucleon, the strange "asymmetry", $s(x)-\bar{s}(x)$, in the number densities of strange quarks and antiquarks, $x$ being the light-cone momentum fraction they carry, is of particular interest. Since the nucleon does not carry any strangeness quantum number, the integral of the asymmetry over all values of $x$ has to vanish:

$$
\langle s-\bar{s}\rangle=\int_{0}^{1} d x[s(x)-\bar{s}(x)]=0 .
$$

However, there is no symmetry that would prevent the $x$ dependences of functions $s(x)$ and $\bar{s}(x)$ from being different. One therefore can expect $s(x) \neq \bar{s}(x)$, in general.

To understand and quantify the strangeness asymmetry in the nucleon is interesting in various different contexts. Some models of nucleon structure make predictions [2, 3, 4, 5, 6, 7, 8] for $s(x)-\bar{s}(x)$, and their confrontation with experimental measurements may perhaps give us further insight into non-perturbative dynamics of the strong interactions. For example, within the meson cloud model [2, 4, 7], contributions to the strange sea arise from fluctuations in the proton wave function to $\Lambda K$ and $\Sigma K$ states. The $\Lambda$ (which contains the $s$ quark) will primarily carry the larger fraction of the nucleon momentum, and one thus expects $s(x)$ to be larger than $\bar{s}(x)$ at large $x$, implying the opposite behavior at small $x$. However, a number of subtleties in this picture have been pointed out and discussed in the literature [9]. Light-cone models [5], on the other hand, generically predict $s(x)-\bar{s}(x)<0$ at large $x$. The various models have in common that they predict a fairly small value of the second moment of the strange-antistrange distribution,

$$
\langle x(s-\bar{s})\rangle \equiv \int_{0}^{1} d x x[s(x)-\bar{s}(x)],
$$

usually $|\langle x(s-\bar{s})\rangle| \sim 10^{-4}$.

As was emphasized in [10, 11], the question concerning the strange asymmetry in the nucleon becomes particularly relevant in view of the "anomaly" seen by the NuTeV collaboration in their measurement of the Weinberg angle in deeply-inelastic neutrino scattering. The NuTeV result [12],

$$
\left.\sin ^{2} \theta_{\mathrm{W}}\right|_{\mathrm{NuTeV}}=0.2277 \pm 0.0013_{(\text {stat. }} \pm 0.0009_{(\text {sys. }},
$$

deviates around three standard deviations from the commonly accepted value $\sin ^{2} \theta_{\mathrm{W}}=0.2228 \pm$ 0.0004 [13]. This large difference could be at least partly explained [10, 11] by a positive value of $\langle x(s-\bar{s})\rangle$. Typically, a value $\langle x(s-\bar{s})\rangle \approx 0.005$ would be required if one wanted to attribute the $\mathrm{NuTeV}$ anomaly to the strange asymmetry alone. Note that, if $\langle x(s-\bar{s})\rangle$ is indeed positive and if one assumes that $s(x)-\bar{s}(x)$ has only one node, then the vanishing of $\langle s-\bar{s}\rangle$ implies that $s(x)-\bar{s}(x)$ is positive at large $x$.

The NuTeV Collaboration has itself determined the second moment $\langle x(s-\bar{s})\rangle$ from a lowestorder QCD analysis of neutrino dimuon data [14] and finds a negative value [15]:

$$
\langle x(s-\bar{s})\rangle=-0.0027 \pm 0.0013
$$

Such a value increases the discrepancy in $\sin ^{2} \theta_{\mathrm{W}}$ to a $3.7 \sigma$ effect. The second moment had also been investigated in "global analyses" of unpolarized parton distributions. Ref. [16] reported an 
improvement in the global analysis if the asymmetry $s(x)-\bar{s}(x)$ is positive at high $x$. They found $\langle x(s-\bar{s})\rangle=0.002 \pm 0.0028$ at $Q^{2}=20 \mathrm{GeV}^{2}$ from their best fit. A recent update of this analysis [17], however, reduces the asymmetry significantly. The most recent global QCD fit [18] finds a large uncertainty for the strange asymmetry and quotes a range $-0.001<\langle x(s-\bar{s})\rangle<$ 0.004 .

The discussion reported so far regards strange-antistrange asymmetries that do not depend on the hard-scattering scale $Q$ at which the nucleon is probed. In this letter, we point out that perturbative QCD definitely predicts a non-vanishing and $Q$-dependent value of the strangeantistrange asymmetry. We will show that non-singlet DGLAP evolution of the parton densities at three loops generates a strange asymmetry even if it is not present at the input scale for the evolution. Thus, we can provide a prediction for the strange asymmetry $s(x)-\bar{s}(x)$ based solely on perturbative QCD. The effect arises because at that order of perturbation theory the probability of a splitting $q \rightarrow q^{\prime}$ becomes different from that of $q \rightarrow \bar{q}^{\prime}$, and because the nucleon has $u$ and $d$ valence densities. The three-loop splitting functions have very recently been published [19, 20], among them the splitting function needed for our perturbative estimate of $s(x)-\bar{s}(x)$. To begin with, we write down the evolution equations and determine the solution for the generated strange asymmetry. We then present some numerical results for the strange asymmetry and extend the analysis to the heavy-quark sector.

\section{Non-singlet evolution equations and their solutions}

The parton distributions $f_{a}\left(x, Q^{2}\right)\left(a=q_{i}, \bar{q}_{i}, g\right)$ of the nucleon evolve according to the evolution equation

$$
\frac{d f_{a}\left(x, Q^{2}\right)}{d \ln Q^{2}}=\sum_{b} \int_{x}^{1} \frac{d z}{z} P_{a b}\left(\frac{x}{z}, \alpha_{\mathrm{S}}\left(Q^{2}\right)\right) f_{b}\left(z, Q^{2}\right),
$$

where $P_{a b}$ is the function describing the splitting $b \rightarrow a$. The splitting functions are perturbative; their perturbative series starts at $\mathcal{O}\left(\alpha_{\mathrm{S}}\right)$ :

$$
P_{a b}=\left(\frac{\alpha_{\mathrm{S}}}{4 \pi}\right) P_{a b}^{(0)}+\left(\frac{\alpha_{\mathrm{S}}}{4 \pi}\right)^{2} P_{a b}^{(1)}+\left(\frac{\alpha_{\mathrm{S}}}{4 \pi}\right)^{3} P_{a b}^{(2)}+\mathcal{O}\left(\alpha_{\mathrm{S}}^{4}\right)
$$

Keeping just the first term yields the leading order (LO) evolution. Improving the approximation by taking into account also the second, or the second and third, terms corresponds to next-toleading order (NLO) and next-to-next-to-leading order (NNLO) evolution, respectively.

It is convenient to introduce Mellin moments of the parton densities and splitting functions,

$$
f_{a}^{N}\left(Q^{2}\right) \equiv \int_{0}^{1} d x x^{N-1} f_{a}\left(x, Q^{2}\right)
$$

so that momentum-fraction convolutions as in Eq. (5) reduce to true products and the evolution equation becomes simply

$$
\frac{d f_{a}^{N}\left(Q^{2}\right)}{d \ln Q^{2}}=\sum_{b} P_{a b}^{N}\left(\alpha_{\mathrm{S}}\left(Q^{2}\right)\right) f_{b}^{N}\left(Q^{2}\right) .
$$

In the following we drop the dependence on the moment index $N$ for simplicity. 
Since $s(x)-\bar{s}(x)$ is a flavor non-singlet (NS) quantity, we only need to consider the NS sector of evolution. In the following we write the evolution kernels $P_{a b}$ by adopting the notation of Ref. [19]. Owing to charge conjugation invariance and and flavour symmetry of QCD, one has (see e.g. Ref. [21])

$$
\begin{aligned}
& P_{q_{i} q_{k}}=P_{\bar{q}_{i} \bar{q}_{k}}=\delta_{i k} P_{q q}^{V}+P_{q q}^{S} \\
& P_{q_{i} \bar{q}_{k}}=P_{\bar{q}_{i} q_{k}}=\delta_{i k} P_{q \bar{q}}^{V}+P_{q \bar{q}}^{S} .
\end{aligned}
$$

The splitting functions $P_{q q}^{S}$ and $P_{q \bar{q}}^{S}$ thus describe splittings in which the flavor of the quark changes. As will become clear below, the effect we wish to investigate originates from the fact that $P_{q q}^{S} \neq P_{q \bar{q}}^{S}$ starting from NNLO [21, 22].

In the flavour NS sector the evolution equations (8) are diagonalized by properly introducing NS combinations of parton densities. Up to NLO it is sufficient to consider two NS combinations. Owing to the difference between $P_{q q}^{S}$ and $P_{q \bar{q}}^{S}$, starting from NNLO it is necessary [22] to introduce the following three NS combinations of parton densities:

$$
f^{(V)} \equiv \sum_{i=1}^{N_{f}}\left(f_{q_{i}}-f_{\bar{q}_{i}}\right) \quad, \quad f_{q_{i}}^{( \pm)} \equiv f_{q_{i}} \pm f_{\bar{q}_{i}}-\frac{1}{N_{f}} \sum_{j=1}^{N_{f}}\left(f_{q_{j}} \pm f_{\bar{q}_{j}}\right)
$$

where $N_{f}$ is the number of flavors. Each of these evolves as

$$
\frac{d \ln f^{(A)}\left(Q^{2}\right)}{d \ln Q^{2}}=P^{(A)}\left(\alpha_{\mathrm{S}}\left(Q^{2}\right)\right), \quad(A=V, \pm),
$$

where the evolution kernels are

$$
P^{(V)}=P_{q q}^{V}-P_{q \bar{q}}^{V}+N_{f}\left(P_{q q}^{S}-P_{q \bar{q}}^{S}\right), \quad P^{( \pm)}=P_{q q}^{V} \pm P_{q \bar{q}}^{V} .
$$

The equations have the solutions

$$
f^{(A)}\left(Q^{2}\right)=U^{(A)}\left(Q, Q_{0}\right) f^{(A)}\left(Q_{0}^{2}\right),
$$

where $f^{(A)}\left(Q_{0}^{2}\right)$ is the parton density at the starting scale $Q_{0}$ and the evolution operator $U^{(A)}$ is given by

$$
U^{(A)}\left(Q, Q_{0}\right)=\exp \left\{\int_{Q_{0}^{2}}^{Q^{2}} \frac{d q^{2}}{q^{2}} P^{(A)}\left(\alpha_{\mathrm{S}}\left(q^{2}\right)\right)\right\} .
$$

Using Eq. (13) with $A=-$ and $A=V$, we have

$$
\left(f_{q_{i}}-f_{\bar{q}_{i}}\right)\left(Q^{2}\right)=U^{(-)}\left(Q, Q_{0}\right)\left(f_{q_{i}}-f_{\bar{q}_{i}}\right)\left(Q_{0}^{2}\right)+\frac{1}{N_{f}}\left(U^{(V)}\left(Q, Q_{0}\right)-U^{(-)}\left(Q, Q_{0}\right)\right) f^{(V)}\left(Q_{0}^{2}\right) .
$$

We remind the reader that the parton distributions as well as the evolution operators depend on the Mellin moment $N$.

\section{Strange-quark asymmetry}

Equation (15) is the basic result in our discussion of flavour asymmetries. The key point is that Eq. (15) implies that, in the region of $Q^{2}$ where QCD perturbation theory is applicable, the flavour 


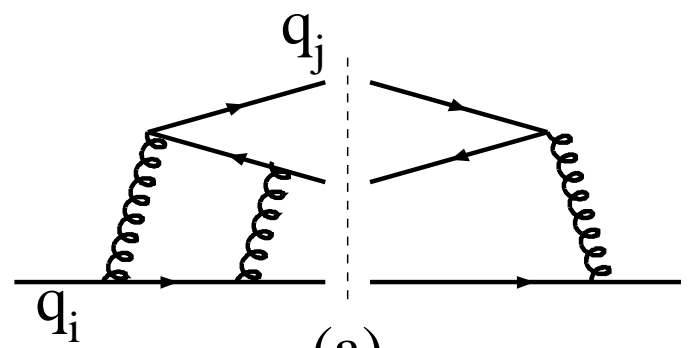

(a)

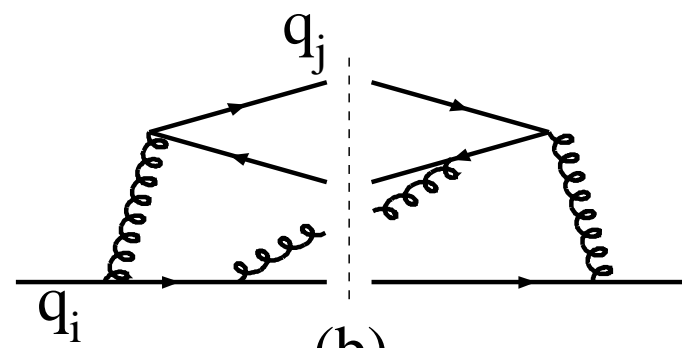

(b)

Figure 1: Example of (a) virtual and (b) real diagrams contributing to $P_{n s}^{(2) S}$

asymmetries $\left(f_{q_{i}}-f_{\bar{q}_{i}}\right)\left(x, Q^{2}\right)$ must necessarily be different from zero (they can vanish, at most, at a single value of $Q$ ). This is a definite, though qualitative, prediction of perturbative QCD.

In the following we simplify the notation, using $f_{q_{i}} \equiv q_{i}$ and $f_{\bar{q}_{i}} \equiv \bar{q}_{i}$, and we consider in detail the strange-quark asymmetry, $s-\bar{s}$. Equation (15) gives

$$
(s-\bar{s})\left(Q^{2}\right)=U^{(-)}\left(Q, Q_{0}\right)\left[(s-\bar{s})\left(Q_{0}^{2}\right)+\frac{1}{N_{f}}\left(\frac{U^{(V)}\left(Q, Q_{0}\right)}{U^{(-)}\left(Q, Q_{0}\right)}-1\right) f^{(V)}\left(Q_{0}^{2}\right)\right] .
$$

At LO and NLO, $U^{(V)}=U^{(-)}$, and thus any strange-quark asymmetry can only be produced by a corresponding asymmetry at the input scale $Q_{0}$ of the evolution. Starting from NNLO, the degeneracy of $P^{(V)}$ and $P^{(-)}$is removed:

$$
P^{(V)}-P^{(-)}=N_{f}\left(P_{q q}^{S}-P_{q \bar{q}}^{S}\right) \equiv\left(\frac{\alpha_{\mathrm{S}}}{4 \pi}\right)^{3} P_{n s}^{(2) S}+\mathcal{O}\left(\alpha_{\mathrm{S}}^{4}\right)
$$

where $P_{n s}^{(2) S}$ has recently been presented in Ref. [19]. It comes with the color structure $d^{a b c} d_{a b c}$, which is also new at this order. In a physical gauge, the Feynman diagrams contributing to $P_{n s}^{(2) S}$ are of the "light-by-light" scattering type, three gluons connecting the two different quark lines. Figure 1 shows some examples of (interferences of) (a) virtual and (b) real diagrams that generate the asymmetry in the evolution of quarks and antiquarks. The virtual part (e.g. Fig.11(a)) has separately been studied [23] in the context of its contribution to the one-loop triple collinear splitting function. When the evolved quark $q_{j}$ is replaced by the antiquark $\bar{q}_{j}$, the abelian-like part of the diagrams in Fig. 1 changes sign, because of the charge asymmetry produced by the exchange of three gluons (vector bosons) in the $t$-channel. This effect occurs both in QCD and $\mathrm{QED}$, and it is a genuine quantum (due to interferences and loop contributions) phenomenon.

The full expressions for $P_{n s}^{(2) S}$ in $N$ and $x$ space may be found in Eqs. (3.9) and (4.11), respectively, of Ref. [19]; also a simple approximation of the function in $x$ space is provided which is sufficiently accurate for our purposes:

$$
\begin{aligned}
P_{n s}^{(2) S}(x) \approx & N_{f}\left(\left[L_{1}\left(-163.9 x^{-1}-7.208 x\right)+151.49+44.51 x-43.12 x^{2}+4.82 x^{3}\right][1-x]\right. \\
& \left.+L_{0} L_{1}\left[-173.1+46.18 L_{0}\right]+178.04 L_{0}+6.892 L_{0}^{2}+40 / 27\left[L_{0}^{4}-2 L_{0}^{3}\right]\right),
\end{aligned}
$$

where $L_{0}=\ln x, L_{1}=\ln (1-x)$. The Mellin moments of this expression are straightforwardly derived and involve only simple harmonic sums [19]. The first moment of the above parameterization is zero to high accuracy. 
On account of Eqs. (16)-(18), even if $(s-\bar{s})\left(Q_{0}^{2}\right)=0$, a non-vanishing strange-quark asymmetry is produced just by the perturbative QCD evolution. Here it is crucial that the total valence density of the nucleon, $f^{(V)}$, is non-vanishing due to the up and down valence quarks. Using Eqs. (14) and (17) we have, in moment space,

$$
\begin{aligned}
\frac{U^{(V)}\left(Q, Q_{0}\right)}{U^{(-)}\left(Q, Q_{0}\right)}-1 & =\int_{Q_{0}^{2}}^{Q^{2}} \frac{d q^{2}}{q^{2}}\left(\frac{\alpha_{\mathrm{S}}\left(q^{2}\right)}{4 \pi}\right)^{3} P_{n s}^{(2) S}+\mathcal{O}\left(\mathrm{N}^{3} \mathrm{LO}\right) \\
& =-\frac{1}{8 \pi b_{0}} P_{n s}^{(2) S}\left[\left(\frac{\alpha_{\mathrm{S}}\left(Q^{2}\right)}{4 \pi}\right)^{2}-\left(\frac{\alpha_{\mathrm{S}}\left(Q_{0}^{2}\right)}{4 \pi}\right)^{2}\right]+\mathcal{O}\left(\mathrm{N}^{3} \mathrm{LO}\right)
\end{aligned}
$$

where

$$
b_{0}=\frac{1}{12 \pi}\left(11 C_{A}-2 N_{f}\right)
$$

and we have used the renormalization group equation

$$
\begin{aligned}
\frac{d \ln \alpha_{\mathrm{S}}\left(q^{2}\right)}{d \ln Q^{2}} & =\beta\left(\alpha_{\mathrm{S}}\left(q^{2}\right)\right) \\
\beta\left(\alpha_{\mathrm{S}}\right) & =-b_{0} \alpha_{\mathrm{S}}-b_{1} \alpha_{\mathrm{S}}^{2}-b_{2} \alpha_{\mathrm{S}}^{3}+\mathcal{O}\left(\alpha_{\mathrm{S}}^{4}\right) .
\end{aligned}
$$

Note that despite being a NNLO effect, the perturbative generation of $s-\bar{s}$ is a leading effect since it first occurs at this order. This explains why only the coefficient $b_{0}$ needs to be taken into account in Eq. (19). Under the assumption $(s-\bar{s})\left(Q_{0}^{2}\right)=0$, and neglecting heavy quark (valence)contributions and threshold effects, the solution for the evolution equation for the strange-quark asymmetry reads to NNLO:

$$
\begin{aligned}
(s-\bar{s})_{N}\left(Q^{2}\right) & =-\frac{P_{n s, N}^{(2) S}}{8 \pi b_{0} N_{f}}\left[\left(\frac{\alpha_{\mathrm{S}}\left(Q^{2}\right)}{4 \pi}\right)^{2}-\left(\frac{\alpha_{\mathrm{S}}\left(Q_{0}^{2}\right)}{4 \pi}\right)^{2}\right] U_{N}^{(-)}\left(Q, Q_{0}\right) f_{N}^{(V)}\left(Q_{0}^{2}\right) \\
& =-\frac{P_{n s, N}^{(2) S}}{8 \pi b_{0} N_{f}}\left[\left(\frac{\alpha_{\mathrm{S}}\left(Q^{2}\right)}{4 \pi}\right)^{2}-\left(\frac{\alpha_{\mathrm{S}}\left(Q_{0}^{2}\right)}{4 \pi}\right)^{2}\right]\left(u^{(V)}+d^{(V)}\right)_{N}\left(Q^{2}\right),
\end{aligned}
$$

where we have restored the Mellin moment index $N$. In the second line we have used that $U_{N}^{(-)}\left(Q, Q_{0}\right)$ simply evolves the valence input, $u^{(V)}=u-\bar{u}$ and $d^{(V)}=d-\bar{d}$, to the scale $Q$ at LO accuracy. Note that, assuming isospin symmetry, the sum of valence distributions is the same in the proton and the neutron and, consequently, also the perturbative strange asymmetry.

\section{Numerical estimates}

From Eq. 23) we can straightforwardly obtain predictions for $[s-\bar{s}]\left(x, Q^{2}\right)$ by a numerical Mellin inversion, once we have chosen an initial scale and input valence densities. For our estimates we employ the low input scale $Q_{0}=0.51 \mathrm{GeV}$ and the $u, d$ valence densities of the LO "radiative" parton model analysis of Ref. [24]. Threshold effects at $Q=m_{c} \equiv 1.4 \mathrm{GeV}$ and $Q=m_{b} \equiv 4.5$ $\mathrm{GeV}$ are taken into account by the full implementation of Eq. (16). Since we are considering a leading effect, the LO approximations are appropriate. The value for the initial scale is of course crucial for our results; the lower the scale, the larger will the perturbatively generated strange asymmetry be at a given higher scale $Q$. Our choice of a rather low input scale may 
be regarded as yielding the largest possible perturbative strange asymmetry. Whether or not it is indeed correct to assume that the nucleon is symmetric in $s$ and $\bar{s}$ at a low scale is an open question; however, our motivation is to explore the new effect provided by three-loop evolution. We note that our input assumption $[s-\bar{s}]\left(x, Q_{0}^{2}\right)$ is consistent with the input in Ref. [24], where actually $s\left(x, Q_{0}^{2}\right)=\bar{s}\left(x, Q_{0}^{2}\right)=0$ was assumed, resulting in a purely generated (symmetric) strange distribution which agrees reasonably well with the ones obtained in other global analyses of PDFs $\uparrow$.

Fig. [2(a) shows $[s-\bar{s}]\left(x, Q^{2}\right)$ as a function of $x$, for three different scales, $Q^{2}=2,10,100 \mathrm{GeV}^{2}$. For comparison, Fig. 2 (b) shows the ratio of $[s-\bar{s}]\left(x, Q^{2}\right)$ to the MRST[25] strange density $s\left(x, Q^{2}\right)$. One can see that the generated asymmetry is not negligible and turns out to be positive at small $x$ and negative at large $x$. Since the distribution has a vanishing first moment and only one node, a negative second moment results:

$$
\langle x(s-\bar{s})\rangle \approx-5 \times 10^{-4} \quad\left(Q^{2}=20 \mathrm{GeV}^{2}\right) .
$$

This value depends fairly little on $Q^{2}$ once $Q^{2}>1 \mathrm{GeV}^{2}$; it then very gently decreases at large $Q^{2}$. As expected for a NNLO effect, it is quite small, somewhat smaller than the NuTeV value quoted in Eq. (4). We also note that our value lies in the band for the second moment derived from a global analysis in Ref. [18].

Let us now try to put the size of the perturbatively generated strange asymmetry into a better perspective. As we discussed above, the effect becomes possible for the first time at NNLO, where $P_{n s}^{(2) S} \neq 0$. This is reminiscent of a well-known effect that first arises in NLO evolution, namely the perturbative generation of $[\bar{u}-\bar{d}](x) \neq 0$ from evolution, due to $P_{q \bar{q}}^{(V)} \neq 0$ at NLO [26]. Interestingly, despite being a NNLO effect, we found our $[s-\bar{s}]\left(x, Q^{2}\right)$ to be larger than the NLO perturbative $[\bar{u}-\bar{d}]\left(x, Q^{2}\right)$ in most of the $x$ region, in particular at small $x$ where the splitting function $P_{n s}^{(2) S}$ is singular as $\ln ^{4} x$ (see Eq. (18) ) [18]. Also, for the perturbative $\bar{u}-\bar{d}$ the difference $\left[u^{(V)}-d^{(V)}\right]\left(x, Q_{0}^{2}\right)$ of input valence densities determines the boundary condition, whereas for $s-\bar{s}$ it is their sum, according to Eq. (23). From this point of view, the perturbatively generated $[s-\bar{s}]\left(x, Q^{2}\right)$ can actually be considered as quite large. Of course, as it is well known, a much larger $\bar{u}-\bar{d}$ asymmetry than the perturbatively predicted one has been measured [27, 28, 29. 30. 31], which implies that non-perturbative effects outweigh the asymmetry from perturbative evolution. It is clearly possible that also in the case of $s-\bar{s}$ non-perturbative effects dominate. It is also worth pointing out that the uncertainties in the perturbative strange asymmetry itself are difficult to quantify since it is effectively a LO effect. On the other hand, as we mentioned in the introduction, models of nucleon structure generally predict a very small strange asymmetry, the second moment usually being several times smaller than ours in Eq. (24). Therefore, at the very least, we expect perturbative evolution to play a significant role in relating model predictions at the (low) model scale to $s-\bar{s}$ at scales relevant for comparison to experimental data.

We also note that the large- $x$ behavior of our perturbatively generated strange asymmetry is driven by that of the evolved valence densities and of the splitting function $P_{n s}^{(2) S}(x)$. As $x \rightarrow 1$, the latter behaves as

$$
P_{n s}^{(2) S}(x) \sim(1-x) \ln (1-x)+\mathcal{O}(1-x),
$$

which because of the convolution with the evolved valence densities implies that

$$
[s-\bar{s}]\left(x, Q^{2}\right) \sim(1-x)^{2} \ln (1-x)\left[u^{(V)}+d^{(V)}\right]\left(x, Q^{2}\right) .
$$

\footnotetext{
ฯThis agreement supports the assumption that the asymmetry should also vanish at a low $Q_{0}$
} 


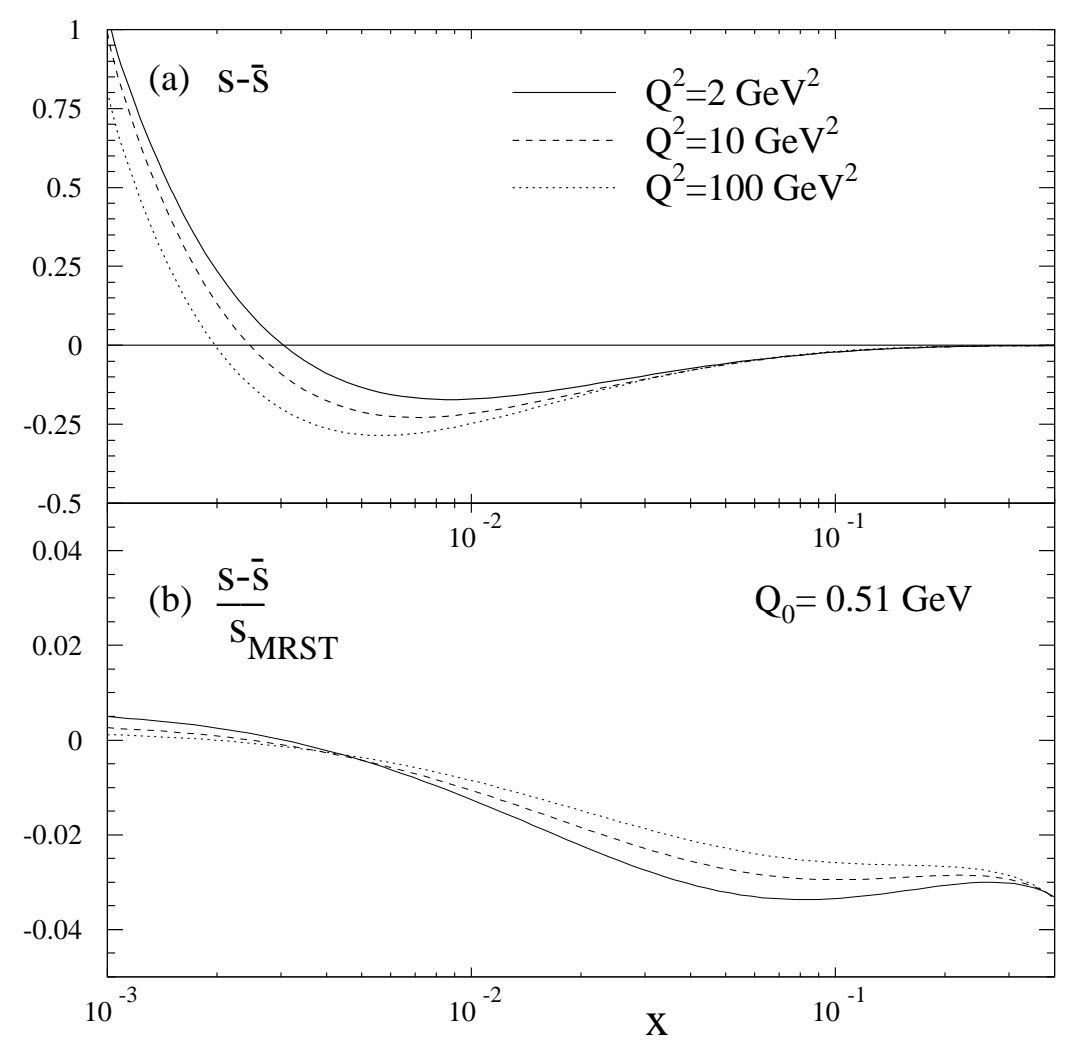

Figure 2: (a) Strange asymmetry in the nucleon generated from NNLO QCD evolution for $Q^{2}=$ $2,10,100 \mathrm{GeV}^{2}$ and (b) the corresponding ratio to the LO strange distribution from Ref. [25]

This may well be the dominant behavior at high $x$, even in the presence of a non-perturbative input distribution for $s-\bar{s}$.

We finally note that our analysis may also be extended to predict the asymmetries for heavy flavors $c$ and $b$. Here, the perturbative prediction may be more reliable since one will typically start the evolution from the mass of the heavy quark, which is in the perturbative region. Assuming that the charm and bottom densities vanish at the respective masses, we find the results shown in Fig. 3 The upper plot (a) compares the purely perturbatively generated charm and bottom densities to the results of the latest MRST LO global analysis [25]. The agreement found at scales far away from the threshold for heavy quark production, and in the relevant small $x$ range, is a signal of the validity of the approach. One can expect that a similar situation holds for the asymmetry. The lower plot (b) corresponds to the ratio between the generated asymmetry and the corresponding heavy quark density. Note that for the last result we only assume that the heavyflavor asymmetries vanish at the respective masses. This is a weaker assumption than there be no initial heavy-quark distributions at all at these scales. As can be observed, the asymmetries are smaller than in the strange sector, mostly due to the larger initial scale at which the evolution begins. 


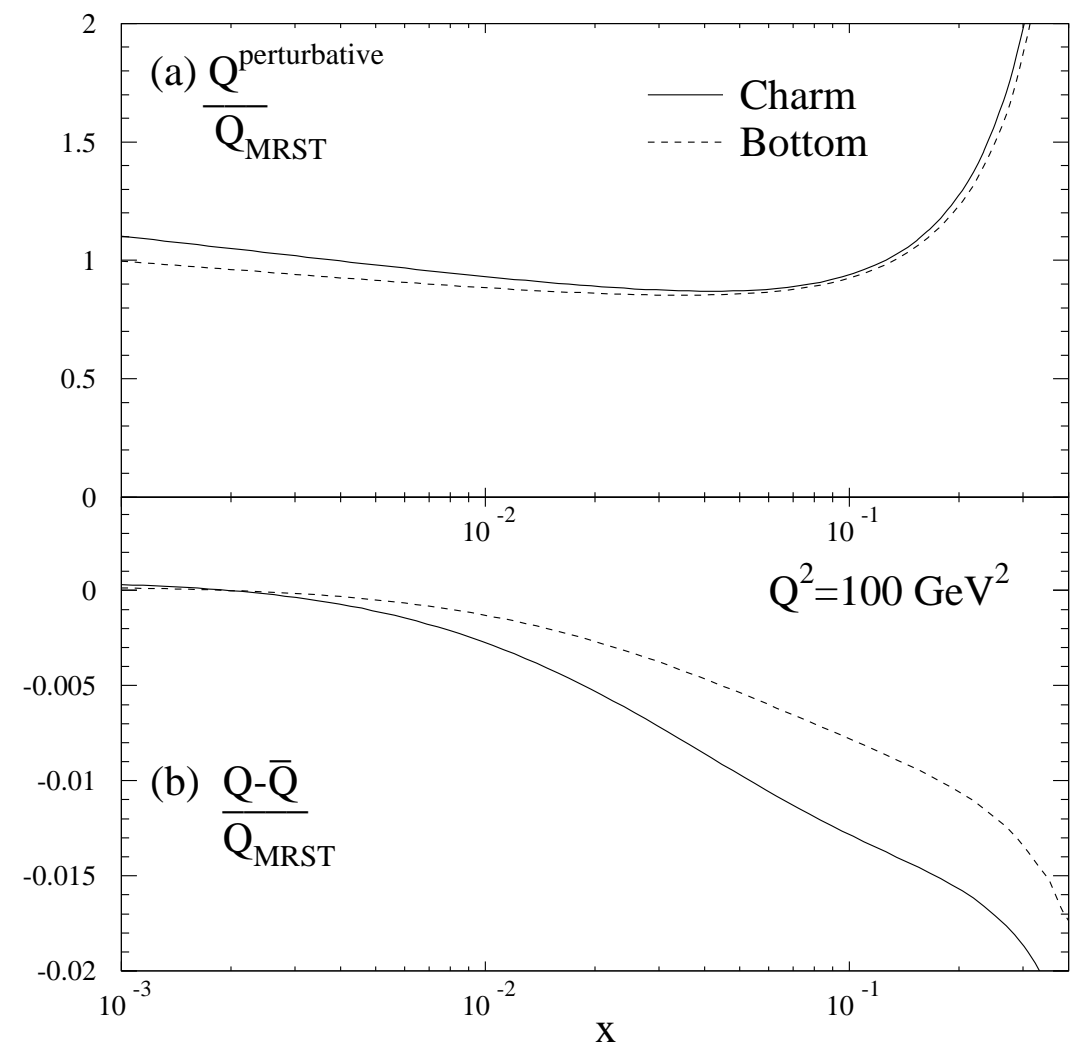

Figure 3: (a) Ratios between the purely pertubatively generated charm and bottom densities at $Q^{2}=100 \mathrm{GeV}^{2}$ to the corresponding distribution from Ref. [25] and (b) (Normalized) charm and bottom asymmetries in the nucleon generated from NNLO QCD evolution.

\section{Acknowledgments}

We are grateful to G. Altarelli and S. Kretzer for useful discussions. G.R. was supported by the Generalitat Valenciana under grant GRUPOS03/013, and by MCyT under grant FPA-2001-3031. The work of D.dF has been partially supported by Conicet, Fundación Antorchas, UBACyT and ANPCyT. W.V. is grateful to RIKEN, Brookhaven National Laboratory and the U.S. Department of Energy (contract number DE-AC02-98CH10886) for providing the facilities essential for the completion of his work.

\section{References}

[1] J. R. Ellis, Nucl. Phys. A684 (2001) 53 [arXiv:hep-ph/0005322].

[2] A. I. Signal and A. W. Thomas, Phys. Lett. B 191 (1987) 205.

[3] M. Burkardt and B. Warr, Phys. Rev. D 45 (1992) 958. 
[4] H. Holtmann, A. Szczurek and J. Speth, Nucl. Phys. A 596 (1996) 631 [arXiv:hep-ph/9601388].

[5] S. J. Brodsky and B. Q. Ma, Phys. Lett. B 381 (1996) 317 [arXiv:hep-ph/9604393].

[6] H. R. Christiansen and J. Magnin, Phys. Lett. B 445 (1998) 8 [arXiv:hep-ph/9801283].

[7] F. G. Cao and A. I. Signal, Phys. Rev. D 60 (1999) 074021 arXiv:hep-ph/9907297.

[8] W. Melnitchouk and M. Malheiro, Phys. Lett. B 451 (1999) 224 [arXiv:hep-ph/9901321].

[9] F. G. Cao and A. I. Signal, Phys. Lett. B 559 (2003) 229 [arXiv:hep-ph/0302206].

[10] S. Davidson, S. Forte, P. Gambino, N. Rius and A. Strumia, JHEP 0202 (2002) 037 [arXiv:hep-ph/0112302].

[11] S. Kretzer, F. Olness, J. Pumplin, D. Stump, W. K. Tung and M. H. Reno, preprint BNLNT-03/16 [arXiv:hep-ph/0312322].

[12] G. P. Zeller et al. [NuTeV Collaboration], Phys. Rev. Lett. 88 (2002) 091802 [Erratum-ibid. 90 (2003) 239902] [arXiv:hep-ex/0110059].

[13] K. Hagiwara et al. [Particle Data Group Collaboration], Phys. Rev. D 66 (2002) 010001.

[14] M. Goncharov et al. [CCFR and NuTeV Collaboration], Phys. Rev. D 64 (2001) 112006 [arXiv:hep-ex/0102049].

[15] G. P. Zeller et al. [NuTeV Collaboration], Phys. Rev. D 65 (2002) 111103 [Erratum-ibid. D 67 (2003) 119902] [arXiv:hep-ex/0203004].

[16] V. Barone, C. Pascaud and F. Zomer, Eur. Phys. J. C 12 (2000) 243 [arXiv:hep-ph/9907512].

[17] B. Portheault, talk at DIS2003, St. Petersburg, 23-27 April 2003.

[18] F. Olness et al., preprint MSU-HEP-030701 [arXiv:hep-ph/0312323].

[19] S. Moch, J. A. M. Vermaseren and A. Vogt, preprint DESY-04-047 [arXiv:hep-ph/0403192]

[20] A. Vogt, S. Moch and J. A. M. Vermaseren, preprint NIKHEF-04-004 [arXiv:hep-ph/0404111].

[21] W. Furmanski and R. Petronzio, Z. Phys. C 11 (1982) 293.

[22] S. Catani and F. Hautmann, Nucl. Phys. B 427 (1994) 475 [arXiv:hep-ph/9405388.

[23] S. Catani, D. de Florian and G. Rodrigo, Phys. Lett. B 586, 323 (2004) [arXiv:hep-ph/0312067].

[24] M. Gluck, E. Reya and A. Vogt, Eur. Phys. J. C 5 (1998) 461 [arXiv:hep-ph/9806404].

[25] A. D. Martin, R. G. Roberts, W. J. Stirling and R. S. Thorne, Eur. Phys. J. C 28, 455 (2003) [arXiv:hep-ph/0211080].

[26] D. A. Ross and C. T. Sachrajda, Nucl. Phys. B 149 (1979) 497. 
[27] R. S. Towell et al. [FNAL E866/NuSea Collaboration], Phys. Rev. D 64 (2001) 052002 [arXiv:hep-ex/0103030]. E. A. Hawker et al. [FNAL E866/NuSea Collaboration], Phys. Rev. Lett. 80 (1998) 3715 [arXiv:hep-ex/9803011].

[28] P. Amaudruz et al. [New Muon Collaboration], Phys. Rev. Lett. 66 (1991) 2712. M. Arneodo et al. [New Muon Collaboration], Phys. Rev. D 50 (1994) 1.

[29] A. Baldit et al. [NA51 Collaboration], Phys. Lett. B 332 (1994) 244.

[30] K. Ackerstaff et al. [HERMES Collaboration], Phys. Rev. Lett. 81 (1998) 5519 [arXiv:hep-ex/9807013].

[31] P. L. McGaughey et al., Phys. Rev. Lett. 69 (1992) 1726. 